\title{
Empiric Versus Resistance-Guided Therapy in Obese Patients with Helicobacter Pylori Infection
}

\author{
Rute Cerqueira ${ }^{1}$ (iD \\ Published online: 12 August 2019 \\ (C) Springer Science+Business Media, LLC, part of Springer Nature 2019
}

\section{Dear Editor}

The Maastricht V/Florence Guideline [1] recommends two empirical Helicobacter pylori (HP) therapy before susceptibility guided therapy (see Statement 15).

Statement 15: After failure of second-line treatment, culture with susceptibility testing or molecular determination of genotype resistance is recommended in order to guide treatment.

After failure of a second-line strategy, treatment should be guided by AST, whenever possible. Resistance to clarithromycin, levofloxacin or rifabutine has a major negative impact on the results of triple therapies. Resistance to metronidazole has a less marked negative effect. Susceptibility-guided triple therapies proved more effective than empirical triple therapies in first-line treatment. In a systematic review, benefits of tailored treatment in second-line treatment remain uncertain, and there is no comparative data for third-line treatment. In most of these studies, strains were only tested for clarithromycin susceptibility. There are no data comparing empirical with susceptibility guided sequential therapy. However, an optimal efficacy of a genotype resistance-guided sequential therapy in third-line treatment of refractory $H$. pylori infection has been reported.

Therefore, two HP empiric therapies are not only accepted but recommended by Maastricht consensus in all patients, obese or not.

Rute Cerqueira

rute.cerqueira@chedv.min-saude.pt

1 Gastroenterology, Centro Hospitalar entre Douro e Vouga, Santa Maria Feira, Portugal

Publisher's Note Springer Nature remains neutral with regard to jurisdictional claims in published maps and institutional affiliations.

Everywhere, most of the patients, like our obese patients group, are managed in a real life scenario. Outside of experimental scenarios is not possible, due to economic and time and logistic constraints (only investigations lab make this test) to perform « ad initio» the sensitivity test or genotype resistance molecular determination.

The Maastricht guidelines author's were certainly aware of these limitations and therefore made a realistic guideline widely applicable.

Compliance with Ethical Standards

Conflict of Interest The authors declare that they have no competing interests.

\section{References}

1. Malfertheiner P, Megraud F, O'Morain CA, et al. Management of Helicobacter pylori infection-the Maastricht V/Florence Consensus Report. Gut. 2017 Jan;66(1):6-30. https://doi.org/10.1136/gutjnl2016-312288. 